
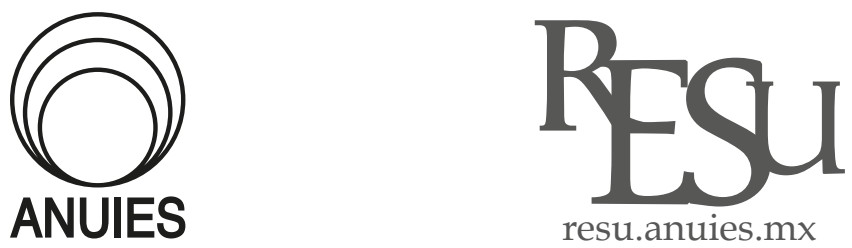

ARTíCULO

\title{
Factores que influyen en la incorporación de las Tecnologías de la Información y la Comunicación en universidades públicas: una aproximación desde la autopercepción docente
}

Factors influencing incorporation of Information and Communication

Technologies in public universities: approach based on teacher selfperception

\author{
Beatriz Zempoalteca*, Juan González ${ }^{* *}$, Jorge Barragán*** y Teresa Guzmán \\ * Universidad Autónoma de Querétearo. Correo electrónico: beatriz.zempoalteca@gmail.com \\ Recibido el 13 octubre del 2017; aceptado el 18 de junio del 2018.
}

\section{Resumen}

La incorporación de las Tecnologías de la Información y la Comunicación (TIC) aún no se percibe consolidada en la praxis pedagógica en las Instituciones de Educación Superior (IES). La presente investigación identifica factores que influyen en la incorporación de las TIC tomando en consideración la autopercepción de docentes adscritos a carreras del área de Ciencias Administrativas de IES públicas. El estudio fue realizado por medio de un método cuantitativo-descriptivo correlacional, con una técnica de encuestas. Se encontró que existen factores internos y externos que favorecen la incorporación de las nuevas tecnologías por parte de la docencia, que resultan de interés para el desarrollo de políticas institucionales sobre la formación del profesorado en las TIC.

Palabras clave: Práctica docente, Tecnologías de la Información y la Comunicación (TIC), Educación Superior Pública, Formación de profesores, Nuevas tecnologías 


\begin{abstract}
The incorporation of Information and Communication Technologies (ICT) has not yet been consolidated in pedagogical practice in Higher Education Institutions (HEI). The present study identified factors that influence the incorporation of ICT taking into consideration the self-perception of teachers assigned to career in the area of business management sciences. The research performed here corresponds to a quantitative-descriptive correlation method, and it has been done through a survey methodology. It was found that there are internal and external factors that favor the incorporation of new technologies by teachers. This is of interest for HEI to develop their ICT strategies for teacher education.
\end{abstract}

Keywords: Teaching practice, Information and communication technologies (ICT), Higher Education, Teacher education, New technologies.

\title{
Introducción
}

L a incorporación de las Tecnologías de la Información y la Comunicación (TIC) en las Instituciones de Educación Superior (IES) aún no se percibe consolidada para su uso en el proceso de enseñanza-aprendizaje, y mucho menos se puede hablar de un uso pertinente de las tecnologías y de su incorporación a la didáctica (Organización de las Naciones Unidas para la Educación, la Ciencia y la Cultura [UNESCO], 2013). Una prueba de ello la exponen Zubieta, Bautista y Quijano (2012), pues mencionan que en la Universidad Nacional Autónoma de México (UNAM), a pesar de los altos niveles de intención de uso de las TIC, la frecuencia de uso es baja, así como también lo son la variedad de aplicaciones y el ambiente Web utilizado en la práctica académica. Un caso similar se da en la Universidad Autónoma de Querétaro (UAQ); allí, de acuerdo a Guzmán, García, Espuny y Chaparro (2011), el uso de las TIC en la práctica docente (cuando se da), se efectúa principalmente en ambiente Web 1.0, el cual expone información unidireccional que implica escasa participación del usuario final como aportador de contenidos, al contrario de otros ambientes dinámicos como la Web 2.0 que permite el intercambio, la aportación y la colaboración (Peñalosa, 2013).

Por otra parte, las IEs públicas en México han centrado más su atención en la adquisición de equipos tecnológicos fijos y de uso personal. En la Agenda Digital Nacional (ADN, 2011), se muestra que en el rubro "infraestructura”, el indicador se incrementó de 0.38 en el año 2005 a 0.48 al año 2010; mientras 
que en el rubro "preparación de personas" descendió de 0.68 en el año 2005 a 0.61 al año 2010, lo cual se ve reflejado en los esfuerzos de las universidades mexicanas orientadas más hacia la infraestructura TIC, que en la formación de las personas, pues, por ejemplo 91\% de las IEs cuenta con un centro de cómputo propio, mientras que $40 \%$ promueve planes de formación en tecnología (Asociación Nacional de Universidades e Instituciones de Educación Superior [ANUIES], 2016). Así, De Pablos (2009) señala como una de las principales problemáticas de esta introducción masiva del uso de las TIC, es aquella que muestra una cierta deformación del propio concepto de cambio educativo, pues se ha concedido mayor peso al equipamiento que a otros aspectos esenciales, tales como la preparación de los docentes.

En este sentido, Bates y Sangrà (2012) mencionan que la introducción de las TIC en la enseñanza, particularmente en el nivel superior, supone un reto importante para la formación del profesorado, pues no se tienen formación ni experiencia suficientes en esta materia; por tanto, las intervenciones al respecto hasta la fecha han sido aisladas, fragmentadas y en ocasiones ineficaces, y han tenido pocas consecuencias positivas en la docencia universitaria, lo cual ha inhibido la participación innovadora (Zenteno y Montera, 2011). Aunado a lo anterior, en México, los docentes de más de 45 años en asignaturas específicas y programas multidisciplinarios conforman alrededor del 50\% del personal activo (Secretaría del Trabajo y Previsión Social e Instituto Nacional de Estadística y Geografía [STPS-INEGI], 2016), el cual, de acuerdo a datos del INEGI (2016), es el segmento de población que tiende a utilizar menos las TIC, pues sólo 18.4\% hace uso de dichas tecnologías. En cambio, los jóvenes de entre 18 y 34 años de edad son los que más las utilizan (representan $37.3 \%$ ). De este modo, se podría entender de manera somera, el bajo uso de las TIC por parte de los docentes, y al mismo tiempo, la amplia brecha digital que existe con los estudiantes universitarios.

De lo antes planteado, resalta la necesidad de llevar a cabo investigaciones que orienten la integración de las TIC en las IES, como puede ser nuestra aproximación, que tiene como objetivo identificar factores que influyen en la incorporación de las TIC en las IES públicas, considerando la autopercepción de los docentes, la cual resulta de gran relevancia para identificar elementos que faciliten la incorporación de la tecnología de una forma efectiva, así como también para diseñar políticas institucionales para que la formación en TIC se efectúe acorde a las necesidades, puesto que los docentes son actores principales de la presencia y de la aplicación que las nuevas tecnologías tienen y pueden llegar a tener en las IEs (Salinas, 2008; Area, 2009; Kounenou et al., 2015; unESCO, 2015; Tárraga-Mínguez et al., 2017; Ruiz y Hernández, 2018). Lo anterior se justifica en parte por el hecho de que al momento no existe una 
certeza de cuáles son los principales factores que influyen en la incorporación de las TIC y cómo se relacionan con su uso en el contexto de la docencia.

Con relación a lo antes citado, en la presente investigación se ha seleccionado específicamente la percepción de docentes adscritos a carreras del área de ciencias administrativas, pues existen hallazgos - en algunos estudios que muestran que los docentes en dichas áreas tienden a utilizar menos las TIC (Muñoz y González, 2012; Zubieta et al., 2012). Asimismo, de acuerdo al INEGI (2014) 42.2\% de los docentes ocupados en el nivel superior en México pertenece a instituciones con carreras de áreas de ciencias administrativas. Por ello, es prioritario que los docentes, en el área referida, utilicen las Tic de manera efectiva, de acuerdo con el contexto y adecuándolas a sus propias formas y maneras de concebir el mundo y de intervenirlo (Morales, Monje y Loyola, 2004; Cabrera, 2006; Sagástegui, 2006; Brun, 2011), además de que es necesario fortalecer las habilidades digitales y establecer estrategias para consolidar la capacitación docente en TIC (Estrategia Digital Nacional [EDN], 2013; Organización para la Cooperación y el Desarrollo Económicos [OCDE], 2014).

\section{Incorporación de las TIC en las IES}

En la actualidad, las TIC en México constituyen mayoritariamente una herramienta para transferir y acceder tanto a la información como a la comunicación (INEGI, 2016); sin embargo, con el cambio acelerado y las exigencias internacionales, las TIC se están convirtiendo en una herramienta necesaria en el proceso global de enseñanza-aprendizaje que el medio requiere, lo que implica la necesidad de diseñar estrategias más amplias para la implementación de la tecnología y que incluya la formación de todas las personas involucradas en el proceso de enseñanza-aprendizaje de las IES, simultáneamente al desarrollo de la infraestructura tecnológica, administrativa o informática (Bates y Sangrà, 2012).

Desde esta perspectiva, la incorporación de las TIC en las IES es un proceso complejo que va más allá de dotar tecnológicamente a las universidades, pues existe una serie de variables críticas, como la existencia de un proyecto institucional, disponibilidad de materiales digitales, cualificación técnica y pedagógica del profesorado, su formación y la predisposición favorable hacia las TIC (Cabero, 2005; Area, 2009; Zenteno y Mortera, 2011).

En este sentido se han realizado grandes esfuerzos por actualizar el sistema educativo al integrar diversas tecnologías en el aula, tendientes a generar habilidades digitales en docentes y estudiantes en el proceso de enseñanzaaprendizaje. No obstante, los resultados aún no son significativos debido a 
diferentes factores que van desde lo económico hasta la falta de motivación hacia el uso de las TIC (De Pablos, 2009; Quintero, Parra y Araujo, 2011; Zenteno y Mortera, 2011; Parra, Gómez y Pintor, 2014).

\section{Las TIC en la práctica pedagógica y en la formación docente}

Organizar y gestionar los espacios educativos para incorporar las herramientas tecnológicas exige un proceso de reconceptualización del entorno de aprendizaje, pues involucra componentes técnicos, estructurales, psicológicos, estratégicos y de gestión (Telem, 1996). Actualmente, dichas funciones son solamente formuladas por tecnólogos y existe poca participación de los docentes o del personal administrativo de la educación, y aun cuando se les da participación, éstos en muchas ocasiones no cuentan con la visión, con la motivación y con las habilidades suficientes para producir un efecto significativo (Salinas, 2008).

Valverde (2009) menciona que la introducción de las Tic en las aulas muestra este efecto significativo sobre la forma en que los alumnos aprenden y cómo los docentes trabajan; para ello, la primera respuesta de las IEs ha sido crear aulas tecnológicas específicas tales como los laboratorios de cómputo. Sin embargo, también han surgido una gran variedad de soluciones alternas, así como accesos a fuentes de información y recursos didácticos que facilitan al estudiante un mejor aprendizaje y al docente un desarrollo de nuevos roles profesionales como tutoría, evaluación de material didáctico, creación de recursos, etc. Por consiguiente, también es necesario que el docente adquiera nuevas competencias para diseñar situaciones de aprendizaje acordes con las necesidades de los estudiantes y no basta con una simple capacitación en técnicas y procedimientos para la enseñanza, al contrario, se necesita un auténtico cambio en la formación del profesorado (OCDE, 2014; Frola, 2016).

Desde esta óptica, la relación entre la formación docente, la manera de entender la enseñanza y la integración de las viejas o de las nuevas tecnologías en el curriculum escolar, son un tema que ha quedado pendiente, tanto en el aspecto conceptual, como en diversas decisiones, acciones y prácticas (Escudero, 2009). Y esto es así, ya que una de las principales circunstancias que se da en las IES es que la formación docente enfrenta algunos dilemas: por un lado, una profesión base y por el otro, una formación docente sistemática que exige trascender supuestos como "la docencia es una cualidad innata", o que se trata de una "aptitud" por el simple hecho de tener un título profesional 
que involucra los contenidos disciplinares a desarrollar; luego entonces, no se precisa una formación inicial y continua (Hojenberg, 2008).

Adicionalmente, existen múltiples opiniones y especificaciones poco desarrolladas sobre la incorporación de las Tic a la formación docente debido a que no se cuenta con un marco de referencia común (UNESCO, 2016). De esta manera, para López y Chávez (2013), la formación docente en tic debe ser un proceso sistemático de preparación permanente y de desarrollo de competencias, en donde una buena actitud y una buena predisposición por parte de los docentes, y de determinadas condiciones, son fundamentales para crear espacios de enseñanza-aprendizaje acordes al contexto educativo (Kounenou et al., 2015; González, 2017; Ruiz y Hernández, 2018).

Por último, también se han desarrollado modelos específicos sobre la formación del profesorado, en los que el docente conjuga el contenido curricular, la metodología de la enseñanza y el conocimiento de los medios tecnológicos que dispone en su aula, con el objetivo de integrar las TIC de una forma eficaz a la práctica docente (Koehler y Mishra, 2006). No obstante, para desarrollar estos modelos, González (2017) menciona que una parte indispensable del marco conceptual del estudio de esta compleja interacción es el contexto, por lo que, en la investigación que aquí se presenta, se buscó identificar precisamente cómo influye el contexto cuando el docente hace uso de las TIC.

\section{El uso de las TIC por parte de la docencia universitaria}

Las IES están pasando por un periodo de cambio, donde la figura del profesor enfrenta grandes retos para el uso de las TIC, pues como se ha señalado, es un proceso arduo y desafiante que involucra diferentes factores, que pueden ser tanto externos como internos al profesorado y que le facilitan u obstaculizan el uso de la tecnología (González y De Pablos, 2015). En este proceso de incorporación de la tecnología por parte de los docentes, también se pueden observar diferentes etapas de acuerdo al uso (Sanhueza, Ponce de León, Cifuentes y Viñuela, 2009).

Dwyer (1995), menciona cinco etapas: la primera se refiere a la introducción, en la cual el docente conoce los conceptos básicos del uso de las TIC, tiene experiencia nula y señala diversos factores que afectan su uso, principalmente de apoyo técnico o conocimiento de estrategias usando la tecnología; la segunda etapa es la adopción, en donde el docente comienza a utilizar las TIC como complemento a su práctica docente, sin embargo, sigue confiando en sus prácticas tradicionales; la tercera etapa corresponde a la adaptación, 
en la cual el docente es capaz de incorporar las TIC en sus clases habituales y utiliza dicha tecnología para la interacción con los estudiantes; la cuarta etapa concierne a la apropiación, que se caracteriza principalmente por la actitud frente a las tecnologías en la cual el docente aplica diferentes usos y herramientas, razón por la cual demanda mayores acceso y disponibilidad de los recursos TIC; la última etapa se refiere a la invención, en donde la capacidad de innovación y descubrimiento por el uso de las TIC adquiere un dominio mayor en cualquier ámbito y además el docente no sólo utiliza las que conoce, sino que a partir de ellas logra desarrollar nuevas herramientas, combinando tecnologías y buscando nuevas ayudas para lograr una mejora continua.

Así, el uso de la tecnología en la práctica docente es un proceso que implica la actividad del ser humano y su interacción con respecto a objetos tecnológicos en un contexto cultural específico; es decir, no sólo es el conocimiento de la tecnología, sino también la incorporación de las condiciones de acceso y de uso que se produce por la participación de hechos en la actividad que se lleva a cabo con las тIC (Pimienta, 2007; Cobo, 2008; Crovi-Drueta, 2010). De esta manera, la tecnología en uso es el resultado del proceso de apropiación que contempla la evaluación y la adaptación a la práctica cotidiana de los individuos, por lo que incorporar la tecnología trae como resultado que el uso innovador de las TIC llegue a ser en seguida habitual (Prado, Romero y Ramírez, 2009).

\section{Factores que influyen en la incorporación de las TIC en la práctica docente}

En virtud de que el uso de las TIC en las IES es un proceso activo que involucra la interacción de la tecnología con las personas en un contexto específico, entonces el perfil del docente juega un papel importante, pues diversos estudios exponen que los factores vinculados con los docentes son los que influyen más en la integración de las TIC (Inan y Lowter, 2010; Ruíz y Hernández, 2018). En este aspecto, las características del docente que están asociadas al uso de las TIC incluyen atributos cognitivos, profesionales y personales, como la edad, el género, la experiencia docente, el departamento al que se adscriban y las competencias en TIC, así como actitudes, percepciones, creencias e interés hacia la tecnología (Kounenou et al., 2015; Gil-Flores, RodríguezSantero y Torres-Gordillo, 2016).

De este modo, Montes y Ochoa (2006), Prado et al. (2009); Celaya, Lozano y Ramírez (2010), Molina y López (2012), Ochoa, Peña y Crovi-Drueta, (2013), 
Ramírez y Casillas (2014), entre otros, señalan que en la sociedad moderna la incorporación de las Tic a la vida cotidiana, al trabajo y a la educación ha modificado la manera de entender y valorizar el conocimiento; por esta razón, proponen que en la incorporación de las TIC se deben tomar en cuenta el conocimiento, el uso, la formación, la disponibilidad, el acceso, las actitudes y las valoraciones sobre éstas en el ámbito de ocupación. De esta forma, las TIC, en la práctica docente, quedan influidas por factores externos relacionados a variables institucionales e instruccionales (como la institución, la formación docente, el acceso y disponibilidad de tecnología, apoyo TIC y el uso de las TIC) y por factores internos como la edad, el género, el grado académico, la antigüedad en la docencia y el interés de los individuos por el uso de la tecnología.

Se han propuesto iniciativas para la elaboración de indicadores para la medición de la incorporación TIC y así proponer políticas basadas en la evidencia (Pelgrum, 2009). De las principales propuestas destacan las de la Comisión Europea (2009), unESCO (2009) y la Unión Internacional de Telecomunicaciones (UIT, 2010), en donde resalta la evaluación de factores tecnológicos e instruccionales como disponibilidad, acceso a infraestructura, apoyo técnico, formación del profesorado y uso de las TIc. De igual manera, autores como Zubieta et al. (2012), Pedraza, Farías y Lavín (2013), Surej (2015) y Nolasco y Ojeda (2016) han realizado propuestas específicas para la medición, las cuales coinciden con las anteriores adicionando los factores actitud y valoración de utilidad.

Diversos autores coinciden en que la capacitación sobre TIC para su uso en el aula es un factor determinante, empero cuando se habla de autopercepción por parte de la docencia, a decir de ellos, manifiestan que la mayor parte del tiempo aprenden por cuenta propia y no se sienten capacitados para utilizar adecuadamente las TIC y, cuando existe formación, se concentra más en aspectos técnico-instrumentales que en los didáctico-curriculares (Llorente, 2008; Cela-Ranilla et al., 2017; Fremio y Andrade-Rodas, 2017). Hilarión (2014) indica que también influye la rapidez con que avanzan las innovaciones tecnológicas, así como la resistencia al cambio tecnológico por parte de los docentes, la cual varía en función del equipo y de la infraestructura disponible. Según los resultados de González y De Pablos (2015), se extrae que el tiempo y la dedicación para la incorporación de las Tic en el proceso de enseñanza, así como el grado de conocimiento y habilidades con dichas tecnologías son variables que dificultan su integración en el aula y las políticas educativas en los centros escolares.

En estudios realizados en países como Brasil, Costa Rica, Colombia, Ecuador, España y Estados Unidos, en lo que concierne a investigaciones que con- 
templan factores internos y externos, se identificó la edad (Surej, 2015; Echeverría, 2014; Ortiz y Chiluiza, 2014; Pozuelo, 2014; Flores-Lueg y Roig-Vila, 2016) en la que fue posible observar que dicho aspecto es influenciado por la experiencia en la docencia o la experiencia en el uso de la tecnología. También se identificó el factor género (Orellana, et al., 2004; Ruiz y Sánchez, 2010; Ortiz y Chiluiza, 2014; Pozuelo, 2014; Fremio y Andrade-Rodas, 2017), en el cual se percibe que los hombres tienen una actitud más positiva hacia el uso de las TIC, no obstante, también se indica que esta diferencia de género puede disminuir con el tiempo. Otro factor fue el de los años de experiencia en la docencia (Muñoz y González, 2012; Echeverría, 2014; Ortiz y Chiluiza, 2014; Parra et al., 2014; Pozuelo, 2014), en donde se observó que aunque podría ser un elemento conveniente en algunos casos, se torna negativo cuando los docentes se aferran a sus clases tradicionales y existe resistencia al cambio.

También se identificaron factores tales como el grado académico, en el cual existe una clara diferencia en los profesores con mayor grado de estudios, pues tienden a promover mayores cambios con las TIC en su práctica docente (Echeverría, 2014; Pozuelo, 2014; Surej, 2015; Mella, García y Cornejo, 2015; Gil-Flores et al., 2016). Finalmente, se encontraron factores como área de conocimiento, tipo de contratación y horas dedicadas a la docencia (Orellana et al., 2004; Muñoz y González, 2012; Echeverría, 2014; Ortiz y Chiluiza, 2014; Pozuelo, 2014), que podrían ser su influencia positiva o negativa dependiendo de las políticas institucionales.

\section{Metodología}

La metodología propuesta es cuantitativa, pues Lévy (2007) menciona que, debido a la composición socio-técnica y cultural que integra la cultura digital, no es suficiente un tratamiento cualitativo, sino que es preciso abordarla desde una forma sistemática a fin de investigar adecuadamente sus consecuencias. Por ello, se parte de un cuestionario compuesto que recaba información sobre los factores internos y externos, siguiendo las recomendaciones de Boggs, Shore y Shore (2004). Por medio de dicho cuestionario, se obtuvo una aproximación sobre el nivel de incorporación de las TIC en diferentes ámbitos de aplicación por parte de los docentes estudiados a partir de sus propias respuestas, y en relación con los factores internos y externos. Se trata, entonces, de una investigación cuantitativa-descriptiva correlacional, del tipo transeccional. 


\section{Objetivos de la investigación}

El objetivo de esta investigación fue identificar factores que influyen en la incorporación de las Tic y cómo se relacionan con el uso de los docentes que imparten alguna asignatura en IEs públicas de las carreras del área de Ciencias Administrativas de cinco IES públicas, las cuales están ubicadas en la zona metropolitana de la ciudad de Querétaro (México).

\section{Población y muestra}

El estudio se llevó a cabo el primer semestre del año 2015 en una población de 334 docentes de las cinco IEs públicas seleccionadas. El tamaño de la muestra fue determinado mediante el criterio del Teorema del Límite Central, el cual indica una muestra constituida por cien elementos o más, pues con dicha cantidad de muestra se asegura una distribución normal y esto sirve para el propósito de hacer estadística inferencial (Kish, 1995).

La selección se realizó por medio de un muestreo estratificado para tener un modelo proporcional al número de docentes en cada institución y posteriormente, un muestreo aleatorio simple en cada una de las IES. Lo anterior se decidió con el objetivo de tener un mayor grado de precisión, pues existen estratos muy pequeños en donde el hecho de emplear sólo un muestreo aleatorio simple no podría asegurar su selección entre las cinco instituciones seleccionadas (Hernández-Sampieri, Fernández y Baptista (2014).

\section{Instrumento}

Para la recolección de datos se utilizó la técnica de medición por encuesta, tomando como base dos instrumentos, uno elaborado por González, Espuny, De Cid y Gisbert (2011) y otro por Zubieta et al. (2012), mediante los cuales se obtuvieron factores internos relacionados a los docentes, así como factores externos como formación, uso, disponibilidad y acceso a recursos TIC. Estos dos instrumentos que ofrecen datos cuantitativos habían sido anteriormente validados de forma independiente y fueron la base a partir del cual se construyó el cuestionario final usado en esta investigación, también validado por expertos en la materia.

El instrumento considera cuatro secciones y 30 reactivos cerrados: la primera sección se refiere a los datos generales de los docentes encuestados 
(edad, género, grado académico, antigüedad en la docencia, horas frente a grupo, tipo de contrato, institución a la cual se encuentran adscritos y número de estudiantes por periodo escolar); la segunda sección consta de nueve reactivos que se refieren a la percepción de acceso y disponibilidad de los recursos TIC en la práctica docente (uso de equipo, uso de internet, acceso a internet y percepción de apoyo técnico de TIc institucional); la tercera sección consiste en tres reactivos que corresponden a percepciones, actitudes y valoraciones hacia las TIC (percepción de interés, percepción de competencia TIC y actitudes y valoración de utilidad sobre las TIC); y la cuarta sección contiene nueve reactivos que recaban información sobre uso y la formación de las TIC (herramientas TIC conocidas y/o utilizadas en diferentes ámbitos, así como cantidad y frecuencia de cursos en TIC).

\section{Procedimiento de recogida de datos}

Una vez seleccionadas las preguntas de los cuestionarios tomados como referencia, se transcribieron a formato electrónico utilizando la aplicación de formularios de la Suite Google Drive, y el cuestionario resultante fue pilotado para probar su confiabilidad por cada factor considerado, así como las condiciones de la aplicación. Para ello, se aplicó la prueba de Alfa de Cronbach en cada uno de dichos factores (Tabla 1), obteniendo en promedio un valor de .85 que indica una evaluación de muy buena de confiabilidad (GarcíaCadena, 2006).

Tabla 1

Resultados de fiabilidad por factor de la incorporación de las TIC

\begin{tabular}{lcc}
\hline \multicolumn{1}{c}{ Factor de análisis } & $\begin{array}{c}\text { Alfa de } \\
\text { Cronbach }\end{array}$ & $\begin{array}{c}\text { Nivel de } \\
\text { fiabilidad }\end{array}$ \\
\hline Acceso, disponibilidad y apoyo TIC & .80 & Aceptable \\
\hline Actitud y valoración de utilidad hacia las TIC & .96 & Perfecto \\
\hline Uso de las TIC & .87 & Muy bueno \\
\hline Formación en las TIC & .75 & Aceptable \\
\hline Fuente: elaboración propia. & &
\end{tabular}

Posteriormente se seleccionaron a los docentes al azar y de manera proporcional, de acuerdo con las listas existentes en cada una de las cinco instituciones (Ryan, 2013). El cuestionario fue enviado vía correo electrónico en los casos en los que se contaba con la dirección electrónica, dejando deshabilitada la opción de capturar el correo electrónico del destinatario para que se 
registraran las respuestas de manera anónima. En otros casos, se les entregó la liga de acceso al cuestionario de manera personal, y cuando los docentes tenían dudas o se les complicaba acceder al cuestionario, se les proporcionaron las facilidades de equipo y la asesoría personalizada a la liga electrónica.

\section{Análisis de datos}

Después de haber aplicado los cuestionarios se procesaron los datos para su codificación a valores numéricos de acuerdo con sus categorías y a las alternativas de respuesta. Para cada factor considerado se realizó una ponderación a una escala 0 a 100 de acuerdo con los indicadores utilizados en cada variable; para conocer el grado de incorporación de las Tic se utilizó una escala de 0 a 400, pues se deriva de la suma de los cuatro factores que son considerados en la mayoría de las investigaciones, los cuales son: acceso y disponibilidad, uso, formación, actitudes y valoraciones hacia las TIC.

Los datos registrados se transfirieron a un archivo para ser analizados mediante estadística descriptiva e inferencial. Para conocer la correlación del uso de las TIC con cada factor, se calcularon las correlaciones de Pearson a un nivel de significancia de .05. Para identificar la relación de las variables personales con el uso de las TIC y con los niveles de su incorporación en la práctica docente, se realizaron pruebas de Chi-cuadrado a un nivel de significancia de .05. Finalmente, para evaluar la diferencia entre factores se realizaron pruebas de ANOVA a un nivel de significancia de .05.

\section{Resultados}

\section{Análisis descriptivo}

De los datos obtenidos de las 100 encuestas a docentes, se encontró que la muestra está distribuida por $55 \%$ de mujeres y $45 \%$ de hombres, siendo $44 \%$ mayores de 44 años (Figura 1).

\section{Insertar Figura 1.}

En lo referente al grado académico, la muestra se conformó por $22 \%$ con licenciatura, $64 \%$ con maestría y $14 \%$ con nivel doctorado, mostrando que 
las diferentes instituciones se han preocupado por la contratación de los docentes con mayor preparación académica; sin embargo, en referencia a la formación en TIC aún no se tiene formalizada, pues el $68 \%$ de los docentes han aprendido de manera autónoma y el 32\% se ha capacitado de manera formal.

En el rubro de años de experiencia como docentes, el 53\% tiene menos de 10 años y el 47\% tiene 11 años o más de antigüedad, asimismo la experiencia en el uso de las Tic en el ámbito académico, a decir de los docentes ha sido enfocada a la Web 1.0 (Figura 2).

\section{Insertar Figura 2.}

En el tema de actividad adicional a la docencia, 26\% registró no tener ninguna otra actividad, 26\% declaró dar clases en otra institución, 41\% mencionó realizar otra actividad laboral diferente a la docencia, y 7\% indicó realizar trabajos de investigación y/o estudios formales. En el factor tipo de contrato, $30 \%$ refirió tener base y $70 \%$ señaló tener un contrato eventual en la institución.

El tiempo que los profesores se dedican a impartir docencia (en promedio) es de 15 horas por semana; no obstante, las horas impartidas se distribuyen de acuerdo a la Figura 3.

\section{Insertar Figura 3.}

En cuanto a los estudiantes que los docentes atienden por ciclo escolar, 50\% respondió atender a más de 61 estudiantes en promedio (Figura 4).

\section{Insertar Figura 4.}

Para evaluar el nivel de incorporación de las tic en la práctica docente, se seleccionaron los factores en los que coincidieron las referencias utilizadas, así como los factores en los cuales, a decir de los docentes, se podía identificar el uso de las TIC en la práctica cotidiana en el contexto específico de las IES estudiadas. Así, se verifica que el factor con mayor valoración es el de actitud y valoración de utilidad hacia las TIC, y el menor es el uso en el ámbito docente (Tabla 2). 
Tabla 2

Estadística descriptiva de cada factor de la incorporación de las TIC considerado

\begin{tabular}{|c|c|c|c|c|c|}
\hline & 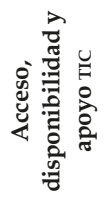 & 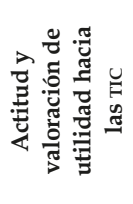 & 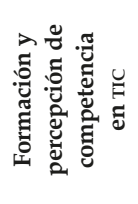 & 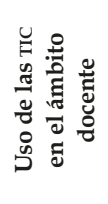 & $\begin{array}{ll} & 0 \\
0 & 0 \\
0 & 0 \\
0 & 0 \\
0 & 0 \\
0 & 0 \\
0 & 0 \\
0 & 0 \\
0 & 0 \\
0 & 0 \\
0 & 0\end{array}$ \\
\hline Media de la muestra estratificada & 62.0 & 83.7 & 49.7 & 34.2 & 65.7 \\
\hline Desviación estándar & 12.9 & 13.5 & 14.6 & 20.3 & 19.6 \\
\hline Error estándar & 1.14 & 1.16 & 1.15 & 1.76 & 1.54 \\
\hline
\end{tabular}

Fuente: elaboración propia.

Con los cuatro factores evaluados, se estimó el nivel de incorporación de las TIC, separando dicha evaluación para el uso en el ámbito docente y para el uso en todos los ámbitos (Tabla 3).

Tabla 3

Estadística descriptiva de los niveles de incorporación de las TIC en la docencia

\begin{tabular}{lcc}
\hline & $\begin{array}{c}\text { Nivel de } \\
\text { Incorporación de } \\
\text { las TIC uso ámbito } \\
\text { docente }\end{array}$ & $\begin{array}{c}\text { Nivel de } \\
\text { Incorporación de } \\
\text { las Tic uso en todos } \\
\text { los ámbitos }\end{array}$ \\
\hline Media de la muestra estratificada & 229.6 & 261.1 \\
\hline Desviación estándar & 43.5 & 42.8 \\
\hline Error estándar & 3.57 & 3.53 \\
\hline Prueba de normalidad Shapiro-Wilk & .991 & .980 \\
\hline p-Valor & p-Valor $>.05$ entonces cumple prueba de normalidad & .141 \\
\hline
\end{tabular}

\section{Análisis correlacional de los factores}

Considerando lo anterior, se estimó la correlación de cada uno de los factores de la incorporación de las TIC, con el uso docente y con el uso en todos los ámbitos (Tabla 3). Se comprobó que el uso tic en la práctica docente se relaciona con la actitud y la valoración de utilidad hacia las tic con $\mathrm{r}=.44(\mathrm{p}<.001)$, lo cual indica que es una dimensión importante, pues a mayor actitud y valoración de utilidad por las TIC, mayor uso en la práctica docente. La correlación de uso de las Tic en todos los ámbitos, obtuvo la correlación más alta con un 
valor de $\mathrm{r}=.49(\mathrm{p}<.001)$ con los tres factores, lo que significa que se combinan todos los factores cuando el uso se lleva a cabo en todos los ámbitos (Tabla 4).

Tabla 4

Correlación entre del uso de las TIC y los factores de actitud, formación y acceso TIC

\begin{tabular}{lcccc}
\hline Correlaciones de Pearson $(r)$ & $\begin{array}{c}\text { Actitud y valoración } \\
\text { de utilidad hacia } \\
\text { las TIC }\end{array}$ & $\begin{array}{c}\text { Formación y } \\
\text { percepción de } \\
\text { competencia en TIC }\end{array}$ & $\begin{array}{c}\text { Acceso, } \\
\text { disponibilidad } \\
\text { y apoyo TIC }\end{array}$ & $\begin{array}{c}\text { Correlación } \\
\text { múltiple }(\boldsymbol{R})\end{array}$ \\
\hline Uso en el ámbito docente & $.44^{*}$ & $.21^{*}$ & $.25^{*}$ & $.44^{*}$ \\
\hline Uso en todos los ámbitos & $.38^{*}$ & $.39^{*}$ & $.34^{*}$ & $.49^{*}$ \\
\hline *probabilidad $(\mathrm{p})<.05$. Sí p $<.05$ entonces relación significativa & & \\
\hline \multicolumn{5}{l}{ Fuente: elaboración propia. }
\end{tabular}

Al clasificar por niveles la incorporación de las TIC y en función de cada uno de sus indicadores, se observa que el nivel 1 (N1) muestra una clara diferencia en la percepción de competencia y de actitud hacia las TIC, siendo el uso informático en ambientes Web 2.0 casi nulo. En el caso del nivel 2 (N2), se distingue un crecimiento considerable en la actitud y la valoración de utilidad hacia las TIC, así como en el nivel de formación formal en TIC, sin embargo, el uso informático en Web 2.0 se mantiene bajo. En lo que compete al nivel 3 (N3), la formación formal en TIC y la frecuencia de uso de internet crece, asimismo el uso en ambiente Web 2.0 se intensifica. Finalmente, en el nivel 4 (N4) se observa una evidente diferencia con respecto a los otros, particularmente en el nivel de formación formal en TIC, en la percepción de acceso a internet institucional, en el uso de equipos y en el uso informático en ambiente Web 2.0, los cuales sobresalen de todos los demás indicadores (Figura 5).

\section{Insertar Figura 5.}

Los niveles de incorporación de las TIC se relacionaron con los factores internos propuestos, y mediante el análisis de Chi-cuadrado se encontró que en la incorporación, considerando el uso docente, se relacionan el grado académico, el número de alumnos y la formación formal en Tic (Tabla 5), en el caso de la incorporación tomando en cuenta el uso en todos los ámbitos, se identificaron la edad, el tipo de contrato, otras actividades adicionales a la docencia y también la formación formal en TIC (Tabla 6). 
Tabla 5

Relación entre los factores y niveles de incorporación de las TIC, uso en el ámbito docente

\begin{tabular}{lccc}
\hline Factor & $\begin{array}{c}\text { Valor Chi-cuadrado de } \\
\text { Pearson }\left(X^{2}\right)\end{array}$ & $\begin{array}{c}\text { Grados de libertad (gl) } \\
n=100\end{array}$ & $\begin{array}{c}\text { Probabilidad } \\
(p)\end{array}$ \\
\hline Edad & 12.98 & 9 & .16 \\
\hline Género & 3.34 & 3 & .34 \\
\hline Institución & 13.73 & 12 & .32 \\
\hline Grado académico & 17.18 & 6 & $<.01$ \\
\hline Antigüedad & 16.67 & 12 & .32 \\
\hline Tipo de contrato & 12.95 & 9 & .16 \\
\hline Horas frente a grupo & 10.66 & 15 & .78 \\
\hline Actividades adicionales & 8.73 & 12 & .76 \\
\hline No. de Alumnos & 16.68 & 9 & $<.01$ \\
\hline Formación formal en tic & 19.19 & 3 & \\
\hline$p<.05$ entonces existe relación significativa. & & \\
$n=$ Tamaño muestral & & & \\
\hline Fuente: elaboración propia. & & & \\
\hline
\end{tabular}

Tabla 6

Relación entre los factores y los niveles de la incorporación de las TIC, uso en todos los ámbitos

\begin{tabular}{lccc}
\hline Factor & $\begin{array}{c}\text { Valor Chi-cuadrado de } \\
\text { Pearson }\left(X^{2}\right)\end{array}$ & $\begin{array}{c}\text { Grados de libertad (gl) } \\
\boldsymbol{n} \mathbf{1 0 0}\end{array}$ & $\begin{array}{c}\text { Probabilidad } \\
(\boldsymbol{p})\end{array}$ \\
\hline Edad & 25.58 & 9 & $<.01$ \\
\hline Género & 4.12 & 3 & .25 \\
\hline Institución & 14.36 & 12 & .28 \\
\hline Grado académico & 7.08 & 6 & .31 \\
\hline Antigüedad & 16.12 & 12 & .19 \\
\hline Tipo de contrato & 17.56 & 9 & .04 \\
\hline Horas frente a grupo & 20.84 & 15 & .14 \\
\hline Actividades adicionales & 23.02 & 12 & .03 \\
\hline No. de Alumnos & 10.55 & 9 & $<.01$ \\
\hline Formación formal en TIC & 31.00 & 3 & \\
\hline$p<.05$ entonces existe relación significativa. & & \\
$n=$ Tamaño muestral & & & \\
\hline Fuente: elaboración propia. & & & \\
\hline
\end{tabular}

Una vez que se identificaron los factores internos relacionados con la incorporación de las TIC, se compararon considerando el uso en el ámbito docente y en todos los ámbitos, en cada uno de los rangos de los diferentes factores (Tabla 7). 
Tabla 7

Nivel de incorporación de las TIC en la docencia por factor

\begin{tabular}{|c|c|c|c|c|}
\hline Factor & No. Datos & Rango & $\begin{array}{l}\text { Nivel de Integración TIC } \\
\text { Uso docente } \\
(M) \\
\end{array}$ & $\begin{array}{c}\text { Nivel de Integración TIC } \\
\text { Uso general } \\
(M) \\
\end{array}$ \\
\hline \multirow{4}{*}{ Edad } & 23 & $<34$ años & 224.1 & 257.1 \\
\hline & 33 & $35-44$ & 212.7 & 246.1 \\
\hline & 31 & $45-54$ & 242.3 & 274.1 \\
\hline & 13 & > 55 años & 232.8 & 255.1 \\
\hline \multirow{2}{*}{ Género } & 55 & Femenino & 226.4 & 255.8 \\
\hline & 45 & Masculino & 228.0 & 261.7 \\
\hline \multirow{3}{*}{ Grado } & 23 & Licenciatura & 204.7 & 241.0 \\
\hline & 63 & Maestría & 231.3 & 261.8 \\
\hline & 14 & Doctorado & 243.4 & 270.5 \\
\hline \multirow{5}{*}{ Antigüedad } & 6 & $<$ un año & 191.2 & 232.0 \\
\hline & 30 & $2-5$ & 228.7 & 259.2 \\
\hline & 16 & $6-10$ & 236.3 & 273.4 \\
\hline & 26 & $11-19$ & 228.6 & 256.2 \\
\hline & 22 & > 20 años & 226.4 & 256.6 \\
\hline \multirow{4}{*}{ Tipo de contrato } & 5 & $\begin{array}{c}\text { Base medio } \\
\text { tiempo }\end{array}$ & 250.1 & 284.1 \\
\hline & 25 & $\begin{array}{l}\text { Base tiempo } \\
\text { comp. }\end{array}$ & 223.5 & 249.2 \\
\hline & 34 & Honorarios & 223.3 & 251.1 \\
\hline & 36 & Asimilados & 230.3 & 267.9 \\
\hline \multirow{4}{*}{$\begin{array}{l}\text { Actividad } \\
\text { adicional a la } \\
\text { docencia }\end{array}$} & 26 & $\begin{array}{c}\text { Otra } \\
\text { universidad } \\
\end{array}$ & 227.7 & 258.7 \\
\hline & 26 & Ninguna & 229.8 & 252.5 \\
\hline & 7 & $\begin{array}{l}\text { Trabajo } \\
\text { académico }\end{array}$ & 240.7 & 268.5 \\
\hline & 41 & Empresa & 222.8 & 260.5 \\
\hline \multirow{4}{*}{$\begin{array}{l}\text { Alumnos por } \\
\text { periodo }\end{array}$} & 8 & $1-20$ & 223.6 & 262.4 \\
\hline & 26 & $21-40$ & 231.7 & 262.8 \\
\hline & 16 & $41-60$ & 246.3 & 270.7 \\
\hline & 50 & $>61$ & 219.2 & 251.7 \\
\hline \multirow{2}{*}{$\begin{array}{l}\text { Formación } \\
\text { formal en TIC }\end{array}$} & 32 & Sí & 256.2 & 292.0 \\
\hline & 68 & No & 213.4 & 242.7 \\
\hline
\end{tabular}

Con referencia a la tabla anterior, se encontró que el factor de formación formal en TIC es el más significativo, pues la prueba de ANOVA muestra que existe diferencia significativa si cuenta o no con formación formal en TIc, además que 
en este rubro se observa la mayor incorporación tic con un valor $M=256.2$ ( $F$ $(1,98)=39.9, p=.001)$. En el caso del factor grado académico se puede observar que el nivel licenciatura es el que presenta el menor nivel de integración de las TIC con un valor $M=204.7(\mathrm{~F}(2,97)=4.44, p=.014)$, el cual aumenta con el nivel de estudios; asimismo, se pueden verificar actividades adicionales a la docencia, particularmente el trabajo académico (como investigación u otros estudios formales) incrementan la incorporación de las Tic en la práctica docente.

\section{Discusión y conclusiones}

De acuerdo a los resultados, se puede identificar que existen diversos factores que se involucran en la incorporación de las TIC en la docencia universitaria, y que no solamente significa incorporar herramientas tecnológicas y formar al profesorado, sino que se debe tratar en forma holística e integradora (Valverde, 2009), pues es un proceso lento y complejo, donde la figura del docente juega un papel preponderante, y en efecto, tiene mucho que ver con las actitudes, con las percepciones y con los intereses de los profesores hacia las TIC, amén de que variables como género y edad pueden resultar irrelevantes cuando el docente tiene una mayor actitud y valoración hacia las TIC (Kounenou et al., 2015; Gil-Flores et al., 2016).

Lo antedicho se comprueba en la evidencia de la importancia de que los docentes sean formados formalmente en el uso de las TIC, pues son éstos los que presentan una mayor integración de dichas tecnologías en su práctica académica. No obstante, en los resultados encontrados, la correlación entre formación en TIC con respecto a su uso es baja, lo cual se debe en gran medida a que actualmente la formación formal es mínima en dichas tecnologías, puesto que la mayoría de los docentes han aprendido el uso de las TIC por medio de autoaprendizaje, y en muy baja proporción se han actualizado de manera constante y con una dirección adecuada, coincidiendo con lo enunciado por Llorente (2008), Fremio y Andrade-Rodas, 2017, Cela-Ranillas et al., (2017).

En referencia al grado académico, también se observó que dicho factor es relevante, pues a mayor grado académico, se observa una mayor integración con las TIC, y esto se puede verificar con las actividades adicionales a la docencia, particularmente con el trabajo académico (como investigación u otros estudios formales), pues existen indicios de que esta actividad incrementa la incorporación de las TIC en la práctica docente, y, por el contrario, no tener ninguna otra, la disminuye, tal como lo observaron Surej (2015), Echeverría (2014) y Pozuelo (2014), quienes perciben una clara diferencia en los profesores con mayor grado de estudios, en cuanto al uso de las TIC en la práctica docente. 
En lo que corresponde a la dimensión de acceso y disponibilidad, se observa que ésta es influenciada de manera positiva por el número de estudiantes menor a 60, pues en la medida que el docente tiene control sobre los recursos TIC percibe un mayor uso; sin embargo, cuando los estudiantes aumentan a más de 60, dicha variable disminuye, pues claramente se prefiere no utilizar las TIC y esto se puede relacionar con lo mencionado por González y De Pablos (2015) en el sentido de que al no contar con tiempo y dedicación por la cantidad de alumnos y/o verse comprometido por la falta de competencia, lo incita a continuar con sus clases tradicionales (Parra et al., 2014).

El factor que tuvo el valor más alto de correlación fue el de actitud y valoración hacia las TIC, en este punto coincide con lo investigado por Orellana et al. (2004), pues de acuerdo con sus resultados, los docentes son conscientes de que las TIC son una herramienta necesaria para el proceso de enseñanza y reconocen su potencialidad, pero esto no se ve reflejado en la práctica docente. En este sentido, se ratificó que el uso de las TIC en la práctica académica aún es muy bajo, además de que sigue predominando el ambiente Web 1.0.

Se encontró también que la antigüedad es un factor decisivo, pues los docentes de nuevo ingreso muestran claramente una falta de formación y de conocimiento de los recursos TIC que puede disponer, lo que ocasiona un nivel de integración muy bajo, no así cuando van adquiriendo mayor experiencia, pues en el rango de 6 a 10 años se encontró la máxima incorporación y posterior a más de 10 años vuelve a disminuir, lo cual corrobora que es importante tomar acciones formativas de mantenimiento para motivar el uso y evitar la monotonía y de esta forma mantener activa la creatividad en el uso de dichos recursos.

En lo que se refiere al factor edad, se identificó que dentro del uso académico, no existe una diferencia significativa, sin embargo, en lo que respecta al rango de 35-44 años, se observa un nivel de integración Tic bajo, que puede deberse a falta de tiempo o interés hacia su uso. Por último, en el factor género se advierte que no existe diferencia de integración en el ámbito docente, no así en el nivel de integración con el uso general, pues se observa una ligera diferencia en el género masculino, como lo identificaron los autores Orellana et al. (2004), Flores-Lueg y Roig-Vila (2016), y Fremio y Andrade-Rodas (2017).

De este modo, sería recomendable promover la capacitación de forma constante y de preferencia obligatoria, no únicamente para garantizar la actualización de los docentes, sino también la inclusión de uso en todo el profesorado, desde una perspectiva pedagógica y sobre todo de sensibilización hacia el cambio en su nuevo rol, como agente crítico y reflexivo de su praxis pedagógica, pues las necesidades en función del contexto cambian y no sólo se trata de conocer las TIC y usarlas, sino que se requiere creatividad para ade- 
cuar las nuevas tecnologías a los requerimientos y situaciones particulares de su materia (Molina y López, 2012; Hilarión, 2014; Falco, 2017; Martínez y Torres, 2017; Ruiz y Hernández, 2018).

\section{Referencias}

Agenda Digital Nacional (ADN) (2011). AMIPCI, AMITI, CANIETI, CIU, Comisión Especial de Acceso Digital de la LxI Legislatura de la Cámara de Diputados y la Comisión de Ciencia y Tecnología de la Lxi Legislatura del Senado de la República. México: ANIEI.

Area, Manuel (2009). Introducción a la tecnología educativa, La Laguna: Universidad de La Laguna.

Asociación Nacional de Universidades de Instituciones de Educación Superior (AnUies) (2016). Estado Actual de las tic en las IEs en México. Estudio ejecutivo 2016. México: ANUIES.

Bates, A.W. (Tony) y Sangrà, Albert (2012). La gestión de la tecnología en la educación superior. Estrategias para transformar la enseñanza y el aprendizaje, Barcelona: Octaedro ICE-UB.

Boggs, Stacey; Shore, Mark y Shore, Joanna (2004). Using e-learning platforms for mastery learning in developmental mathematics courses. Mathematics and Computer Education, 38 (2), 213-220.

Brun, Mario (2011). Las TIC en la formación inicial docente de América Latina. Serie políticas sociales, Naciones Unidas-Santiago de Chile: CEPAL.

Cabero, Julio (2005). Las TIC y las Universidades: retos, posibilidades y preocupaciones. Revista de la Educación Superior, 34 (3), pp. 77-100.

Cabrera, Valeria (2006). Democratización de la Información en Chile. El lugar de la apropiación social de las Tic en la agenda digital 2004-2006. Comunicación y Medios (en línea) 17.

Cela-Ranilla, José-María; Esteve-González, Vanessa; Esteve-Mon, Francesc; González-Martínez, Juan; Gisbert-Cervera, Mercè (2017). El docente en la sociedad digital: Una propuesta basada en la pedagogía transformativa y en la tecnología avanzada. Profesorado. Revista de Currículum y Formación de Profesorado, vol. 21, núm. 1, 2017, pp. 403-422.

Celaya, Rosario; Lozano, Fernando y Ramírez, María-Soledad (2010). Apropiación tecnológica en profesores que incorporan recursos educativos abiertos en educación media superior, Revista Mexicana de Investigación Educativa, 15 (45), pp. 487-513.

Cobo, Cristóbal (2008). Aprendizaje adaptable y apropiación tecnológica: reflexiones prospectivas. México: Flacso México.

Comisión Europea (2009). Indicators on ICT in Primary and Secondary Education. Disponible en: http://eacea.ec.europa.eu/llp/studies/study_on_indicators_ on_ict_education_en.php

Crovi-Drueta, Delia (2010). Acceso, Uso y Apropiación de las tic en comunidades Académicas. Diagnóstico en la UNAM, México: UnAM. 
De Pablos, Juan (2009). La formación del profesorado en la era del Internet, Málaga: Aljibe.

Dwyer, David (1995). Changing the conversation about teaching learning and technology. A report on 10 years of Apple Classrooms of Tomorrow (АСОТ) research. Apple Computer Inc., Cuppertino, C.A., EE.UU.

Echeverría, Ana-Cristina (2014). Uso de las тIc en la Docencia Universitaria: Opinión del Profesorado de Educación Especial. Revista actualidades investigativas en educación, 14 (3) pp. 1-24.

Estrategia Digital Nacional (EDN). (2013). Educación de Calidad. México: Gobierno de la República. Disponible en: http:/ / cdn.mexicodigital.gob.mx/EstrategiaDigital.pdf

Escudero, Juan (2009). Comunidades docentes de aprendizaje, formación del profesorado y mejora de la educación. Ágora, 10, 7-31.

Falco, Mariana (2017). Reconsiderando las prácticas educativas: tics en el proceso de enseñanza-aprendizaje. Tendencias pedagógicas, 29, pp. 59-76.

Flores-Lueg, Carolina y Roig-Vila, Rosabel (2016). Factores personales de estudiantes de pedagogía que inciden en su formación social, ética y legal del uso de тіс. Revista Hipótese, Itapetiniga, 3(3), pp. 3-25.

Fremio, Jhon y Andrade-Rodas, Esteban (2017). Nivel de conocimiento de las TIC en los docentes de educación superior. INNOVA Research Journal, Vol. 2, núm. 12, pp. 59-74.

Frola, Patricia (2016). Maestros competentes a través de la planeación y evaluación por competencias. México: Trillas.

García-Cadena, Cirilo-Humberto (2006). “La medición en las Ciencias Sociales y en Psicología”, en Landeros Hernández, R; \& González Ramírez, M. R. Estadística con SPSS y metodología de la investigación. México: Trillas.

Gil-Flores, Javier; Rodríguez-Santero, Javier y Torres-Gordillo, Juan-Jesús (2016). Factors that explain the use of ICT in secondary-education classrooms: The role of teacher Characteristics and school infrastructure. Computers in Human Behavior, 68, 441-449.

González, Alicia y De Pablos, Juan (2015). Factores que dificultan la integración de las Tic en las aulas. Revista de Investigación Educativa, 33 (2), 401-417.

González, Juan; Espuny, Cinta; De Cid, María-José y Gisbert, Mercè (2012). INCOTIC-ESO. Cómo autoevaluar y diagnosticar la competencia digital en la escuela 2.0, Revista de investigación Educativa, 30 (2), pp. 287-302.

González, Norma (2017). Influencia del contexto en el desarrollo del conocimiento tecnológico pedagógico del contenido (ТРАСК) de un profesor universitario. Virtualidad, Educación y Ciencia, 14 (8), pp. 42-55.

Guzmán, Teresa; García, María-Teresa; Espuny, Cinta y Chaparro, Ricardo (2011). Formación docente para la integración de las TIC en la práctica educativa. Revista apertura (UDG Virtual, México), 3 (1).

Hernández-Sampieri, Roberto; Fernández, Carlos y Baptista, Pilar (2014). Metodología de la investigación, México: Mc Graw Hill.

Hilarión, Leidy (2014). Factores que influyen en la no incorporación de las tic en los procesos de Enseñanza-Aprendizaje por parte de los docentes en la educación superior. Bogotá: Universidad Militar Nueva Granada. 
Hojenberg, Vanesa (2008). ¿A qué jugamos? Un paso por el programa de formación y capacitación docente, Revista Reflexión Académica en Diseño y Comunicación, año IX, vol. 9, pp. 200-204.

Inan, Fethi y Lowter, D. (2010). Laptops in the K-12 classrooms: Exploring factors impacting instructional use. Computer and Education, 55, 937-944.

Instituto Nacional de Estadística y Geografía (INEGI). (2014). Encuesta Nacional de Ocupación y Empleo (ENOE). Cuarto trimestre, 2014.

Instituto Nacional de Estadística y Geografía (INEGI). (2016). Encuesta Nacional sobre disponibilidad y uso de Tecnologías de la Información en los Hogares (ENDUTIH) 2016.

Kish, Leslie (1995). Diseño estadístico para la investigación. Madrid: Centro de Investigaciones Sociológicas.

Koehler, Matthew y Mishra, Punya (2009). What is technological pedagogical content knowledge? Contemporary Issues in Technology and Teacher Education, 9 (1), 60-70.

Kounenou, Kalliope; Roussosb, Petros; Yotsidic, Vasiliki y Tountopoulouc, Maria (2015). Trainee teachers' intention to incorporating ICT use into teaching practice in relation to their psychological characteristics: The case of group-based intervention. Procedia - Social and Behavioral Sciences, 190 (2015) 120-128.

Lévy, Pierre (2007). Cibercultura, informe al Consejo de Europa, ed. $1^{\mathrm{a}}$. Barcelona: Anthropos-UAm.

López, María-Cristina y Chávez, José-Antonio (2013). La formación de profesores universitarios en la aplicación de las тic. Sinéctica, núm. 41, pp. 2-18.

Llorente, María-del-Carmen (2008). Aspectos fundamentales de la formación del profesorado en Tic. Pixel-Bit. Revista de Medios y Educación, 31, pp. 121-130

Martínez, Karla y Torres, Luisa (2017). Estrategias que ayudan al docente universitario a conocer, apropiar e implementar las tic en el aula. Pixel-Bit. Revista de Medios y Educación, 50, pp. 159-172.

Mella, Karina; García, Yonnhatan y Cornejo, Yasna (2017). Factores que afectan la implementación didáctica de algunas tecnologías en el aula. Revista Educación y Tecnología, 7, pp. 74-90.

Molina, Amelia y López, Alma (2012). Formación de profesores indígenas y condiciones para la apropiación de las Tic. Revista Panamericana de Pedagogía, Saberes y Quehaceres del Pedagogo, 19, pp. 57-72, México: Centros culturales de México, A. C.

Montes, Jairo-Andrés y Ochoa, Solanlly (2006). Apropiación de las Tic en cursos universitarios. Acta Colombiana de Psicología, 9 (2), 87-100.

Morales, Susana; Monje, Daniela y Loyola, María-Inés (2004). Apropiación crítica de NTC's como camino de inclusión en la Sociedad de la Información: el caso de los jóvenes de una escuela secundaria de la ciudad de Córdoba. Razón y Palabra (en línea), 52.

Muñoz, Pablo y González, Mercedes (2012). La integración de las Tic en la universidad. Perfiles educativos, vol. xxxIv, núm 137, pp. 46-67.

Nolasco, Pedro y Ojeda, Mario-Miguel (2016). La evaluación de la integración de las TIC en la educación superior: fundamento para una metodología. REDRevista de Educación a Distancia, 48 (9). 
Ochoa, Rafael-Leonardo; Peña, José-Ismael y Crovi-Drueta, Delia-María (2013). Aproximación a un modelo unificador de la difusión de innovaciones tecnológicas al interior de organizaciones. En: 1er Congreso Internacional “Análisis e innovación en las organizaciones", 8 de noviembre de 2013, Mexicali, México.

Orellana, Natividad; Almerich, Gonzalo; Belloch, Consuelo y Díaz, María-Isabel (2004). La actitud del profesorado ante las TIC: Un aspecto clave para la integración. DOI:10.13140/2.1.2264.6089

Organización de las Naciones Unidas para la Educación, la Ciencia y la Cultura (unESCO) (2009). Medición de las TIC en educación-Manual del Usuario. Montreal, Canadá: UNESCO-IEU.

Organización de las Naciones Unidas para la Educación, la Ciencia y la Cultura (UNESCO) (2013). Enfoques estratégicos sobre las TICS en educación en américa Latina y el Caribe. Chile: OREAL/unesco Santiago.

Organización de las Naciones Unidas para la Educación, la Ciencia y la Cultura (UNESCO) (2015). Guía para el desarrollo de políticas docentes. Francia: unESCO-SCI.

Organización de las Naciones Unidas para la Educación, la Ciencia y la Cultura (UNESCO) (2016). Competencias y estándares TIC desde la dimensión pedagógica. Cali: UNESCO-PUJ.

Organización para la Cooperación y el Desarrollo Económicos (OCDE). (2014). Habilidades y competencias del siglo XXI para los aprendices del nuevo milenio en los países de la OCDE. Paris: Instituto de Tecnologías Educativas.

Ortiz, Pedro y Chiluiza, Katherine (2014). Factores y relaciones que afectan la incorporación de TIC en la Educación Superior. Research Gate. Disponible en: https://www.researchgate.net/publication/28797546

Parra, Sandra-Rocío; Gómez, Marcela-Georgina y Pintor, María-Manuela (2014). Factores que inciden en la implementación de las TIC en los procesos de enseñanza-aprendizaje en $5^{\circ}$ de primaria en Colombia, Revista Complutense de Educación, 26 (Especial), pp. 197-213.

Pedraza, Norma; Farías, Gabriela y Lavín, Jesús (2013), Las competencias docentes en TIC en el área de negocios y contaduría, Perfiles Educativos, vol. xxxv, núm. 139, pp. 8-24

Pelgrum, Willem (2009). Indicators on ICT in primary and secondary education: results of an EU study. In Friedrich, S. \& Francesc, P. (Eds.), Assessing the effects of ICT in education (pp. 165-188). Luxembourgo: Publications Office of the European Union.

Peñalosa, Eduardo (2013). Estrategias docentes con tecnologías: guía práctica. México: Pearson.

Pimienta, Daniel (2007). Brecha digital, brecha social, brecha paradigmática. Funredes. Disponible en: http://www.educoas.org/RestrictedSites/Curso1/ Newsletter-Marzo09/Paratenerencuenta42.html

Pozuelo, Jaione (2014). ¿Y si enseñamos de otra manera? Competencias digitales para el cambio metodológico. Revista Caracciolos, Vol. II, núm. 1, pp. 1-21.

Prado, Carlos-Alberto; Romero, Sandra-Irene y Ramírez, María-Soledad (2009). Relaciones entre los estándares tecnológicos y apropiación tecnológica. Revista Enseñanza \& Teaching, 27 (2), pp. 77-101. 
Quintero, Jaider; Parra, Doris y Araujo, Dory (2011). Integración de las TIC en la práctica docente en la Universidad de la Guajira. Revista electrónica de estudios Telemáticos, 10 (1).

Ramírez, Alberto y Casillas, Miguel-Ángel (2014), Háblame de TIC: Tecnología digital en la educación superior, Argentina: Editorial Brujas. Disponible en: http:/ / www.uv.mx/personal/albramirez/2014/08/01/hablamedetic

Ruíz, Julio y Sánchez, José (2010). El género como factor en la estrategia para integrar las TIC en la práctica docente. Pixel-Bit. Revista de Medios y Educación, 37, pp. 67-76.

Ruiz, María-del-Carmen y Hernández, Víctor (2018). La incorporación y uso de las TIC en educación infantil. Un estudio sobre la infraestructura, la metodología didáctica y la formación del profesorado en Andalucía. Píxel-Bit. Revista de Medios y Educación. 52, pp. 81-96.

Ryan, Thomas (2013). Sample Size Determination and Power. Hoboken, New Jersey: Wiley.

Sagástegui, Diana (2006). Uso y apropiación del Programa Enciclomedia en las escuelas primarias de Jalisco. Ix Congreso Nacional de Investigación Educativa, en línea: http://www.comie.org.mx/congreso/memoriaelectronica/v09/ ponencias/at07/PRE1189534811.pdf

Salinas, Jesús (2008). Innovación educativa y uso de las TIC. Sevilla: Universidad Internacional de Andalucía.

Sanhueza, Juan-Alberto; Ponce de León, María-Paulina; Cifuentes, KatherineDaniela y Viñuela, Raúl (2009). Usos, integración curricular y adopción tecnológica de la informática educativa en las prácticas pedagógicas de docentes de La Araucanía, Chile. Revista Iberoamericana de Educación, 49 (5).

Secretaría del Trabajo y Previsión Social y el Instituto Nacional de Estadística y Geografía (stPS-INEGI). (2016). Observatorio Laboral. Panorama de empleo 2016. México: STPS, Disponible en: http:/ / www.observatoriolaboral.gob.mx

Surej, John (2015). The integration of information technology in higher education; a study of faculty's attitude towards IT adoption in the teaching process. Contaduría y Administración. 60 (S1), pp. 230-252.

Tárraga-Mínguez, R.; Sanz-Cervera, P.; Pastor-Cerezuela, G. y Fernández-Andrés, M. I. (2017). Análisis de la autoeficacia percibida en el uso de las TIC de futuros maestros y maestras de educación infantil y Educación primaria. Revista Electrónica Interuniversitaria de Formación del profesorado, 20 (3), pp. 107116.

Telem, Moshe (1996). MIs implementation in schools: A socio-technical Framework. Computers and Education, 27 (2), 85-93.

Valverde, Jesús (2009) Organización educativa de los medios y recursos tecnológicos. De Pablos, Juan. La formación del profesorado en la era del Internet, (pp. 217231). Málaga: Ed. Aljibe.

Unión Internacional de Telecomunicaciones (UIT). (2010). Indicadores clave sobre TIC. Partnership para la medición de las TIC para el desarrollo. Ginebra, Suiza.

Zenteno, Alfredo y Mortera, Fernando-Jorge (2011). Integración y apropiación de las TIC en los profesores y alumnos de educación media superior. Apertura 3 (1).

Zubieta, Judith; Bautista, Tomás y Quijano, Álvaro (2012). Aceptación de las tic en la docencia, México: unAm. 